\title{
Minimal multi-camera system for perception of deformable shapes
}

\author{
Rafael Herguedas, Gonzalo López-Nicolás, Carlos Sagüés \\ Grupo de Robótica, Percepción y Tiempo Real (RoPeRT) \\ Instituto de Investigación en Ingeniería de Aragón (I3A) \\ Universidad de Zaragoza, Mariano Esquillor s/n, 50018, Zaragoza, Spain. \\ Tel. +34-976762707, e-mail: rherguedas@unizar.es
}

\begin{abstract}
Within the context of autonomous manipulation of deformable objects, we propose a minimal multicamera perception system that allows to cover a deforming 2D shape over time according to a specific visibility objective. Our method iteratively solves an optimization problem that includes collision avoidance and robust visibility constraints.
\end{abstract}

\section{Introduction}

Perception of deformation is one of the main concerns when dealing with autonomous manipulation of deformable objects, a topic of increasing interest in the domestic and industrial fields [1]. In this kind of applications, a continuous feedback of the object's deformation is needed, which may be challenging due to the time-varying and highly-dynamic behavior of deformable objects. Thus, we conceive a perception system in the industrial context, where the deformation guidelines are set depending on the object and the particular task. In order to analyze the state of the object and check if the deformation is performed as expected, the system must adapt to the variable object configuration by placing the sensors at the most convenient locations according to the perception requirements. In addition, the number of necessary cameras must be minimized to deal with the fact of having a limited resources availability.

The main property we use for defining how the perception task must be performed is the visibility of the contour. Visibility problems have been addressed from many different perspectives, and here we mainly focus on those where sensors are dynamically deployed in an environment of time-dependent nature. Camera networks from the active perception field are a good example of systems that must reconfigure themselves depending on the environment and the task requirements [2], [3].

This work includes some of the scientific contributions of the Collaborative Robotic Mobile Manipulation of Deformable Objects in Industrial
Applications (CoMManDIA) project. CoMManDIA project aims to improve the competitiveness and working conditions of those industries where deformable objects are manipulated by human operators (more info in http://commandia.unizar.es).

\section{Framework and objectives}

We represent the deformable object as a segmented 2D shape whose contour varies over time. This shape is enclosed by a set of cameras with restricted Field of View (FOV) at a certain safety distance to the object's centroid (see Fig. 1). We assume that the position of the cameras with respect to a global reference is known, as well as the desired contour of the deformable object over time.

Given this framework, the objective is finding the minimal set of sensors that achieve the target visibility, which is defined in terms of the number of sensors that must detect each contour segment, over a complete deformation process.

\section{Multi-camera optimization}

Firstly, in order to define a feasible target visibility we need information not only about the object and the particular deformation task, but also about the maximum achievable visibility, i.e. the maximum number of contour segments that can be detected from a certain distance of the shape. Our method for obtaining this property is based on the bi-partite visibility graph, whose nodes are the vertexes of the contour segments and the optical centers of the cameras. The edges of the graph link the cameras with those vertexes that are visible for them. Then, we check if the two vertexes of each segment are linked to a common camera, and if so we determine that the segment is visible. After knowing the upper bound of segments that can be detected, we are able to define the target visibility and start to solve the optimization problem. This problem is solved with a proposed cost function whose value must be minimized, which includes three different terms. The main term is related to the target visibility error, and the other two prevent the neighboring cameras to be 
closer than a certain threshold distance and introduce robust perception criteria. We apply the pattern search method in an iterative way at each deformation instant, considering an initial set of two cameras and increasing it until the target visibility is achieved. Once the optimal positions and orientations of the minimal set of cameras are obtained, we apply path planning to describe the movement of the cameras along deformation instants.

\section{Simulation results}

We have tested the performance of our method in a series of simulations over contours extracted from the MPEG-7 public dataset. Figure 1 shows the maximum achievable visibility of a triangular shape, in which the $82.2 \%$ of the contour is visible from the safety distance. A deformation process is simulated in Figure 2, and the bi-partite visibility graph of the optimized camera system is presented. Finally, occluding circular obstacles are included in Figure 1 around a bat shape at a single deformation instant.

\section{Conclusions}

By means of graph theory and optimization techniques, we are able to place and orient a minimal set of cameras to cover a 2D shape that deforms over time. First, we compute the maximum achievable visibility of the shape, that allows us to define a feasible target visibility. Then, we solve iteratively the constrained optimization problem until the system achieves the target visibility with a minimal number of sensors. We have performed a series of simulations to analyze the validity of our method and explore future improvements. More details about this work can be found in [4].

\section{Acknowledgments}

The authors would like to thank Leonardo Fermín and Edgar Ramírez for their invaluable help. This work was supported by projects COMMANDIA
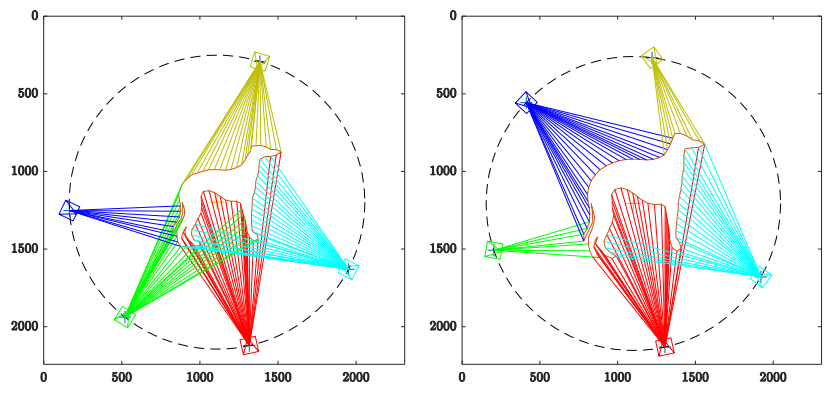

SOE2/P1/F0638 (Interreg Sudoe Programme, ERDF), PGC2018-098719-B-I00 (MCIU/ AEI/ FEDER, UE) and DGA T45-17R (Gobierno de Aragón). The first author was partially supported by the EU through the European Social Fund (ESF) "Construyendo Europa desde Aragón".

\section{REFERENCES}

[1]. SÁNCHEZ, J., CORRALES, J.A., BOUZGARROU, B.C. and MEZOUAR, Y. Robotic manipulation and sensing of deformable objects in domestic and industrial applications: a survey. The International Journal of Robotics Research. 2018, vol. 37, no.7, pp. 688-716.

[2]. PICIARELLI, C., ESTERLE, L., KHAN, A., RINNER, B. and FORESTI, G.L. Dynamic reconfiguration in camera networks: a short survey. IEEE Transactions on Circuits and Systems for Video Technology. 2015, vol. 26, no. 5, pp. 965 - 977.

[3]. NUGER, E. and BENHABIB, B. Multi-camera activevision for markerless shape recovery of unknown deforming objects. Journal of Intelligent \& Robotic Systems. 2018, vol. 92, no. 2, pp. 223-264.

[4]. HERGUEDAS, R., LÓPEZ-NICOLÁS, G., and SAGÜÉS, C. Multi-camera coverage of deformable contour shapes. Submitted 2019.

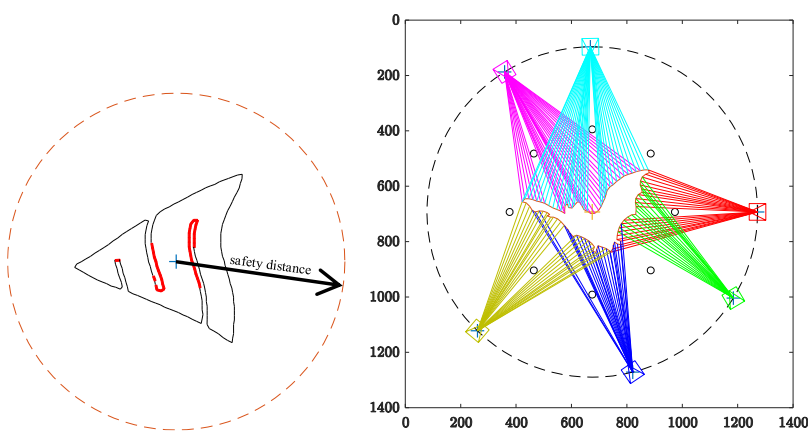

Figure 1. At left, visible (black) and occluded (red) segments for any set of sensors placed at the dashed line circumference around a triangular shape. At right, bipartite visibility graph of a bat shape surrounded by eight occluding obstacles.
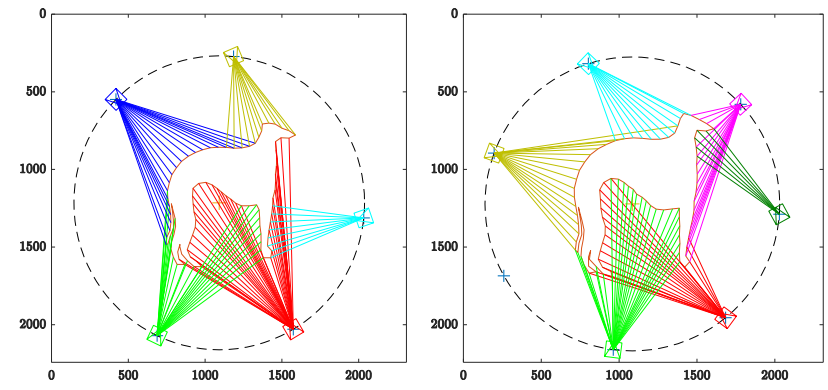

Figure 2. Bi-partite visibility graphs of a complete deformation process. From left to right, a dog shape (orange lines) is increasingly distorted to represent deformation. The minimal number of cameras varies from 5 at the first three deformation instants to 6 at the last one.

Revista “Jornada de Jóvenes Investigadores del I3A”, vol. 7 (Actas de la VIII Jornada de Jóvenes Investigadores del I3A - 6 de junio de 2019). ISSN 2341-4790. 